\title{
Apropiación y uso tecnologías ADS-B en el CETAD
}

\author{
ADS-B Technologies Use and Appropriation at CETAD 2
}

\author{
Jimmy Flórez-Zuluaga ${ }^{3}$
}

\begin{abstract}
CIENCIA Y PODER AÉREO
ISSN 1909-7050 | E-ISSN 2389-2468 | Volumen 9 | Enero-Diciembre de 2014 | Colombia | Pp. 125-134 Recibido: 17/10/2014

Aprobado evaluador interno: 02/11/2014 Aprobado evaluador externo: 18/11/2014
\end{abstract}

${ }^{1}$ Artículo científico original, derivado del proyecto "Sistema de Comando y Control Colombiano (HORUS): Análisis de Transporte y ADS-B", desarrollado por el CETAD.

2 Original research article, product of the project titled as "Colombian System of Command and Control (HORUS): Analysis of Transport and ADS-B", developed by CETAD.

${ }^{3}$ MsC. TIC's. Capitán de la Fuerza Aérea Colombiana, Jefe de Investigación del Centro de Desarrollo Tecnológico Aeroespacial para la Defensa - CETAD. Correo electrónico: florezjimmy@gmail.com

MsC. in Technologies of information and communication. Capitan of the Colombian Air Force, Chief of research at Technological Development Center for Defense-CETAD. E-mail: florezjimmy@gmail.com
Resumen: El transporte aéreo ha venido creciendo en los últimos años, motivo por el cual mantener la seguridad y mejorar el sistema de apoyo a la navegación son retos constantes en el espacio aéreo más congestionado. Una alternativa que ha sido aceptada, a nivel mundial, como ventajosa por sus costos y sus prestaciones, implica la integración de los sistemas satelitales de navegación con sistemas de radiodifusión autónomos en las aeronaves, que han permitido extender la alerta situacional a áreas en las que no se cuenta con cobertura de sistemas CNS/ATM a través de un sistema ADS-B de vigilancia automático dependiente de la aeronave que permite compartir su perfil de vuelo, con aeronaves cercanas y con estaciones de tierra indistintamente. Este sistema se está integrando a los sistemas de vigilancia y control de muchos países, como respuesta a la necesidad de mantener la seguridad aérea en espacios aéreos cada vez más transitados, lo que ha permitido una mejor utilización de las rutas y la disminución de costos operacionales a las empresas. La Fuerza Aérea Colombiana a través del Centro de Desarrollo Tecnológico para la Defensa - CETAD, está trabajando en entender y adaptar esta tecnología para poder explotarla en operaciones militares.

Palabras clave: Predicción de trayectorias, procesamiento de señales radar, tracking radar.

Abstract: Air transport has been growing over the last few years, and for this reason, maintaining the safety and improving navigation systems support is a constant challenge in congested airspace. An alternative that has been accepted worldwide because of its advantageous costs and benefits, involves the integration of satellite navigation systems with autonomous broadcasting systems on aircraft, which have allowed to extend situational awareness into areas without coverage of CNS/ATM systems, through an automatic dependent surveillance aircraft system called ADS-B network which allows flight profile sharing with nearby aircraft and ground stations indifferently. This system is being integrated with the monitoring and control systems of many countries, in response to the need of maintaining the air safety in increasingly trafficked airspaces, allowing better use of routes and decreasing operational costs of companies. The Colombian Air Force, through its Technological Development Center for Defense CETAD, is working on understanding and adapting this technology to utilize it in military operations.

Key Words: Radar Signal Processing, Trajectory Forecasting, Tracking radar. 


\section{Introducción}

La necesidad del ser humano de conocer su entorno y su deseo de expandirse más allá de las fronteras visuales, lo ha obligado a buscar una manera de georeferenciarse, de generar rutas, de navegar a través de la tierra, el mar y el cielo. En un principio buscó el apoyo de los astros, pero con el desarrollo de la tecnología y el pasar de los años, estos fueron reemplazados por sistemas artificiales que orbitan organizadamente la tierra, formando constelaciones completas. Estos sistemas proporcionan muchos servicios entre ellos, servicios de comunicaciones, análisis meteorológico, imágenes, sistemas de ubicación a través del Sistema Global de Navegación por Satélite mejor conocido como GNSS por sus siglas en inglés. Los sistemas de posicionamiento son ampliamente utilizados en investigación, para automatizar procesos y buscar mayores precisiones, como los sistemas de auto navegación inteligente descritos en Chiang y Huang (2008) donde se analiza la aplicación de redes neuronales, combinada con filtros predictivos para mejorar los sistemas el desempeño de los sistemas de posicionamiento.

Otro aspecto de investigación, son los sistemas de colaboración en la navegación aérea, debido a la oportunidad que ofrecen para soportar el crecimiento del transporte aéreo, que mueven miles de personas y miles de millones de dólares anualmente y ha mostrado según los boletines de seguridad operacional de la $\mathrm{OACl}$ un crecimiento del 3.5\% en los últimos años, lo que conlleva a la búsqueda de una administración cada vez más eficiente y segura del espacio aéreo. Los sistemas colaborativos utilizan los servicios globales de navegación que consisten en sistemas pasivos que a través de la triangulación de satélites, logran definir la posición de un cuerpo, para soportar su funcionamiento. El nombre de este servicio es dado por la red de satélites que utiliza el dispositivo para su ubicación, actualmente existen varias redes, entre ellas Global Positioning System (GPS), Glonass, Galileo y Beidou pertenecientes a diferentes países como los Estados Unidos, Rusia, la Unión Europea y China respectivamente.

Los sistemas CNS/ATM(Comunicaciones, Navegación, Vigilancia y Administración del Tráfico Aéreo) fueron creados para garantizar la seguridad y la eficiencia del uso del espacio aéreo, buscando el uso racional de los recursos con estrictas normas de seguridad, como los descritos en Yamamoto (2014) donde se muestran problemas actuales del sistema ATC y posibles alternati- vas, a través de una discusión de expertos de diferentes partes del mundo y el desarrollo de nuevas tecnologías.

Debido a que el control del tráfico aéreo tiene la responsabilidad de mantener la seguridad aérea, el CNS/ATM se constituye como un sistema crítico que debe garantizar la integridad de las aeronaves, para ello actualmente muchos investigadores buscan aplicar nuevas tecnologías examinando sistemas y métodos cada vez más seguros y eficientes como lo descrito en (Flavio y Camargo, 2011) o en el artículo "ADS-X the Next Gen Approach for the Next Generation Air Transportation System" descrito en Harrison (2006) en el que se muestra, cómo la FAA (Federal Aviation Agency), invierte cuantiosas sumas de dinero en el desarrollo de procedimientos experimentales en busca de una nueva generación de sistemas que den mayores prestaciones de seguridad y confiabilidad.

Este artículo pretende mostrar cómo la información de posicionamiento satelital se está integrando en los sistemas ATM a nivel mundial a través de tecnologías como Automatic Dependent Surveillance(ADS), que se perfila como una red de vigilancia cooperativa que responde a las expectativas mundiales de la administración del tránsito aéreo, pero que también se convierte en un nuevo reto para fortalecer y garantizar la seguridad y la independencia del sistema.

El CETAD, como centro de desarrollo de la FAC es consciente de las deficiencias en el sistema de CNS/ ATM en el país y por tal motivo inicio la búsqueda de tecnologías de bajo costo que le permitan a las aeronaves de la FAC realizar operaciones, manteniendo altos estándares de seguridad operacional y que pueda ser integrado con los desarrollos actuales en Comando y Control existentes en el centro. Después de un estudio de prospectiva, se tomó la decisión de iniciar la revisión bibliográfica de los sistemas ADS con el fin de apropiar este tipo de tecnología para la FAC. Después de esta etapa, se realizó una serie de pruebas para comprender el funcionamiento de los dispositivos, sus ventajas y desventajas e iniciar el proceso de apropiación del sistema, proceso que también será abordado en este artículo, donde se describirá el funcionamiento de la tecnología y las pruebas realizadas por el CETAD.

\section{Marco Teórico y estado del arte}

Durante muchos años, los sistemas de tránsito aéreo han usado los radares como principal sensor, para tener 
control de las aeronaves y cómo fuente primaria de información del sistema de vigilancia aérea. Los sensores radar permiten la transducción de la realidad física a señales digitales, las interpreta y las muestra en una pantalla de forma entendible para el controlador. Un sistema de vigilancia y control del espacio aéreo es básicamente aquel que lleva de forma referenciada a una pantaIla, una representación de la realidad tridimensional del espacio aéreo en todo momento. Para poder realizar un adecuado control del espacio aéreo, se conforman redes de sensores de radares, que buscan ampliar la cobertura del sistema de vigilancia (Ver Figura 1). Estas redes deben tener todas las condiciones de sistemas computacionales críticos (Rushby, 1994).

Los radares son sensores que derivan su nombre de las siglas de Radio Detection and Ranging (detección y medición de distancias mediante ondas radioeléctricas), y operan por radiación de energía electromagnética y detección del eco, que retorna producido por la reflexión de la señal en un objeto u objetivo (Skolnik, 1990).

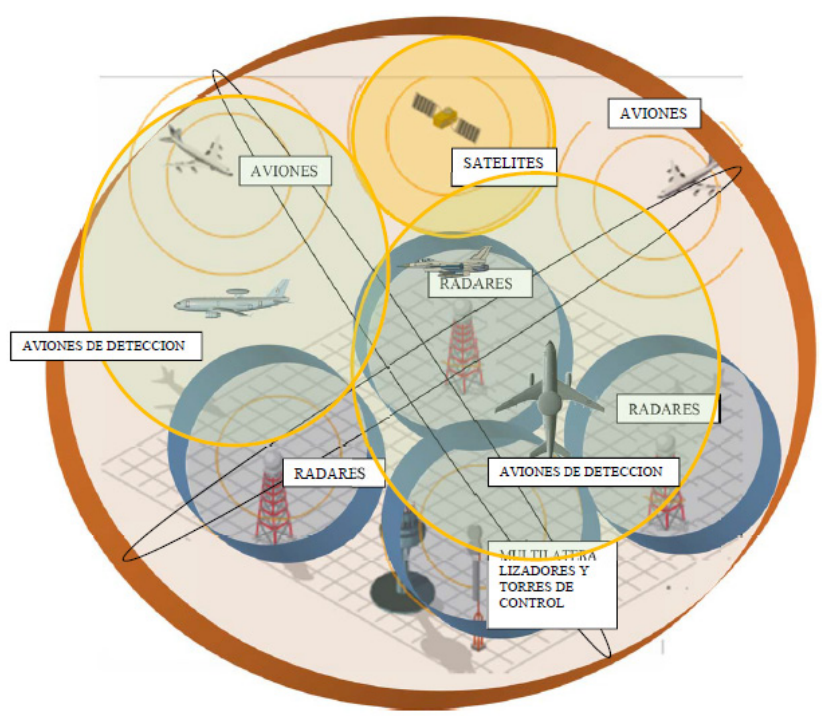

Figura 1. Sistema de ATC.

Fuente: Documentación Sistema Horus.

En el contexto de la vigilancia y control del espacio aéreo se trabaja especialmente con los radares de tipo primario y secundario. Cuando se habla de radar primario se puede generalizar a cualquier sistema que de manera activa realice detecciones, independiente del blanco; el radar secundario en cambio, es un sistema de detección que requiere que el objetivo tenga un dispositivo que genere una señal que es detectada por el receptor. El transmisor de este sistema que va en la aeronave se denomina transpondedor. Normalmente un radar de vigilancia está conformado por la combinación de un sistema radar primario y uno secundario, independientes entre sí con diferentes alcances (Ver Figura 2).

En los radares de control de tránsito aéreo, a diferencia de los radares militares, los rangos de los primarios son mucho menores que los de los secundarios, debido a los costos de operación; todos estos conceptos son ampliados en manuales y referencias de la UAEAC y de la OACl (1998).
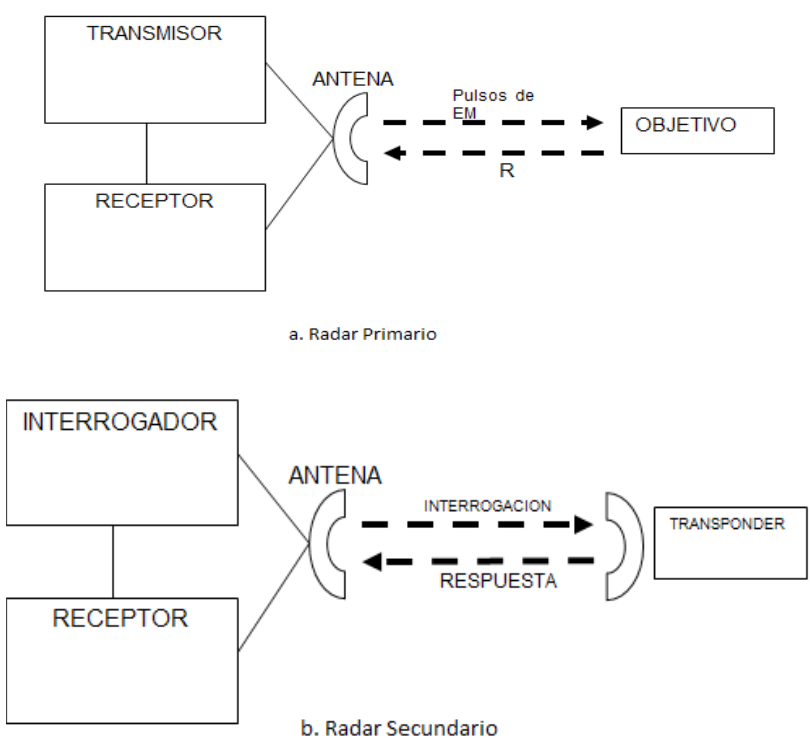

Figura 2. Estructura Radar Primario y Secundario.

Fuente: Documentación CETAD.

Existen dos grandes desventajas en la operación de los radares, la primera son los altos costos de mantenimiento y operación y la segunda es la reducida área de cobertura, debido a la tecnología que utilizan los sensores. Por otro lado, los radares son sistemas independientes de la aeronave, es decir, para mantener la conciencia situacional de las aeronaves en vuelo y garantizar la seguridad debe existir un experto encargado de gestionar el espacio aéreo en mención, denominado controlador de tránsito aéreo, quien a través de sistemas de comunicación, transmite los vectores de posibles aeronaves en conflicto en su área de responsabilidad. Es decir, la información de los radares es solo transmitida a las aeronaves a través de sistemas de comunicación aeronáutica y esta es realizada por una tercera parte; no existe una comunicación entre las aeronaves a través del radar.

La aeronave debe contar también con un piloto que interprete esta información y tome decisiones en cabina. Sin embargo, en los últimos años se ha visto 
un vertiginoso crecimiento de los vuelos no tripulados de acuerdo a datos de Investspain. En seis años, el uso de este tipo de tecnologías a aumentado cuatro veces. Debido al crecimiento de la utilización del espacio aéreo en general, en todo tipo de misiones, tanto por aviones tripulados como por sistemas autónomos, se ha venido trabajando en el desarrollo de una nueva generación de redes CNS/ATM. Esta nueva generación son redes cooperativas de intercambio de información, como se expuso en el International Workshop on ATM/CNS realizado en Tokio en 2009 (Rees, 2009), que pretenden garantizar la administración eficiente y segura del espacio aéreo, que actualmente presenta limitaciones para su control.

La Vigilancia Dependiente Automática (ADS) está siendo desarrollada para solventar estas limitaciones. En esta técnica de vigilancia una aeronave transmite, vía enlace de datos, una serie de parámetros extraídos de los sistemas de navegación y posicionamiento de abordo. La Organización de la Aviación Civil Internacional OACI (2006), define los parámetros para la aplicación de la vigilancia dependiente automática, como un sistema compuesto que comprende un componente abordo y un componente en tierra, lo que lo hace dependiente de la existencia de cierta aviónica. El primero debe suministrar al segundo, datos propios de la navegación de manera automática, con fines de vigilancia. La ADS ha sido desglosada en dos técnicas que están basadas en los mismos principios: ADS-B (Broadcast) y ADS-C (Contract).

En este artículo se desarrollará la aplicación de vigilancia dependiente automática Broadcast (ADS-B), descrita detalladamente en ICAO (2012). Esta técnica consiste en una emisión abierta de información de posición (latitud y longitud), altitud, velocidad, identificación de la aeronave, entre otros datos. Para ello, se vale de información extractada del sistema de navegación de la aeronave y de un posicionamiento satelital. En el caso de que se utilice la información del sistema inercial de la aeronave debe ponerse en la trama una bandera que indique la fuente de la información. Los sistemas más modernos combinan la información del inercial y del GPS de la aeronave para generar la información de posición. Esta información es radiodifundida a intervalos frecuentes y regulares tanto a estaciones en tierra como otras aeronaves con el equipo apropiado, permitiendo la representación del tráfico circundante en una pantalla den- tro del avión, CDTI (Cockpit Display of Traffic Information). Los equipos utilizados para implementar ADS-B son principalmente tres: Modo S Extended squitter, VDL (VHF Data Link) y UAT (Universal Acces Transceiver). Estos sistemas buscan generar la capacidad de vigilancia automática dependiente de la aeronave, como se menciona en Strohmeier, Schafer, Lenders, y Martinov (2014). A diferencia de los sistemas de radar utilizados en Colombia, esta tecnología permite a las aeronaves compartir entre ellas automáticamente sus ubicaciones e incluso su perfil de vuelo, y proporcionar una mayor conciencia situacional en tiempo real, independiente de la cobertura de las estaciones terrenas e incluso llegado el caso, independiente del sistema de ATS, como sucede en áreas que cuentan con pocos recursos tecnológicos de apoyo como el espacio aéreo interoceánico.

Según Schafer, Strohmeier, Lenders, Martinovic, y Wilhelm (2014) en Europa Central ya se cuenta con una red de sensores capaces de procesar el 30\% del tráfico comercial basados en la Red ADS-B, sistema denominado OpenSky. También, se describe en este documento el cambio paradigmático en la forma en la que se realiza la vigilancia del espacio aéreo a nivel mundial, y cómo a través de una red de sensores de bajo costo generar una gran cobertura. Los mensajes ADS-B, son transmitidos por el equipo a bordo de los aviones de manera periódica, y utilizan la banda de $1090 \mathrm{MHz}$, tanto a otras aeronaves como a las estaciones de control en tierra. Actualmente, el mensaje ADS-B es radiodifundido una vez por segundo por cada aeronave para transmitir el vector de estado y otra información de identificación, estos mensajes pueden adoptar una de dos formas, dependiendo de la cantidad de información, un modo básico o corto de 56 bytes o un mensaje largo de 112 bytes.

Durante los últimos años, organizaciones como la $\mathrm{OACl}$ y la FAA vienen realizando esfuerzos para la unificación de criterios y la compatibilidad tecnológica de esa capacidad a nivel mundial como los descritos en ICAO (September, 2011) donde se documenta el procedimiento de implementación del sistema ADS. Sin embargo, se han visto enfrentados cada vez más la exigente necesidad de información del sistema. Según la FAA este tipo de equipos deberá convertirse en obligatorios en la mayoría de los espacios aéreos en los próximos años. Por lo tanto, es importante que sus características y desafíos se investiguen a fondo. 
En Colombia, hay dos organizaciones responsables de garantizar el adecuado uso del espacio aéreo; la Unidad Administrativa especial de la Aeronáutica Civil (UAEAC) que es la encargada de velar por la seguridad en las operaciones aéreas. Es decir, debe garantizar separaciones aéreas entre las aeronaves que vuelan diariamente en el país (Tomlin, 1998; Nolan, 1998). Por otro lado, la Fuerza Aérea Colombiana debe garantizar el uso legal del espacio aéreo y negar el uso a organizaciones al margen de la ley. Ambas trabajan en la integración de sus sistemas de vigilancia y control con la información proveniente del sistema ADS-B.

Tanto la FAC como la UAEAC, soporta sus sistemas de vigilancia y control aéreo en un protocolo denominado ASTERIX, que fue desarrollado por la Agencia Europea para la Seguridad y la Navegación Aérea y son las siglas de "Estructura Multipropósito de Intercambio de Información Radar de Eurocontrol" y su acrónimo corresponde con "All Purpose Structure Eurocontrol Radar Information Exchange". Es un protocolo de capa de aplicación (Capa 6 del modelo OSI, Standard 7498), donde se encuentra definida la estructura de los datos, así como su ensamblaje en formato de mensaje. Fue desarrollado para intercambiar información entre sistemas de vigilancia aérea y entre sistemas de control, tanto civil como militar y es ampliamente utilizado en todo el mundo. El protocolo define también los requerimientos mínimos, para el intercambio de datos entre aplicaciones de diferentes tecnologías. En el estándar, los datos a intercambiar se clasifican en categoría de datos. La mínima unidad de información está conformada por ocho bits denominados data Item. Existen campos específicos que definen la longitud en octetos del Data Block incluidos los campos CAT y LENG y uno o más registros conteniendo información de la misma categoría (Harmonisation y Programme, 2002). En la Figura 3 se aprecia la estructura de un Bloque de datos en una trama Asterix.

En una trama ASTERIX se pueden definir 256 categorías clasificadas en tres familias: aplicaciones estándar civiles y militares, aplicaciones reservadas para uso militar y aplicaciones no estándar civiles y militares. En el sitio web de "Eurocontrol" se puede encontrar la lista de categorías de datos ASTERIX. En Colombia actualmente se está realizando una modernización de los sistemas CNS/ATM con el fin de integrar la tecnología recomendada para la implantación de los sistemas ADS y su interpretación como categoría 21 del ASTE-
Figure 1 - STRUCTURE OF DATA BLOCK OF ASTERIX PROTOCOL
Figura 1 - ESTRUCTURA BLOQLE DE DATOS PROTOCOLO ASTERI

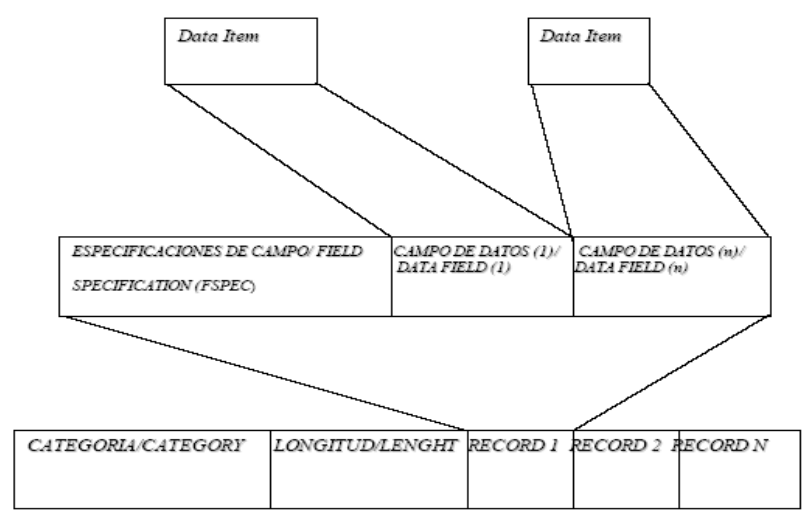

Figura 3. Esquema de Trama Asterix (OACl, 2002).

Fuente: Documentación CETAD.

RIX, para el intercambio de información proveniente de los sensores ADS-B.

Todo esto se integra en un sistema eficiente y seguro donde se reúne el empleo de transpondedores más precisos en los aviones y sistemas de procesamiento y tracking, eficientes como los sustentados en Miquel, Mora-Camino, y Loscos (2006), donde se describen técnicas de control no lineal basado en la linealización por realimentación aplicados al cálculo de las trayectorias de las aeronaves, para flexibilizar de manera segura la administración de los terminales y áreas de control.

\section{EI ADS-B y su integración a la vigilancia del espacio aéreo Colombiano}

En la guía de consideraciones técnicas operacionales para la implantación del ADS-B en la región de Sur América (SAM) que es la que soporta a Colombia, desarrollada en el 2013, (O. R. S. OACl, 2013) y se definen todas las condiciones técnicas a nivel de equipos terrestres y a nivel de equipos abordo que se deben cumplir, con el fin de garantizar la interoperabilidad para todo el continente.

Se define de acuerdo al documento 9924 AN/474 manual de vigilancia aeronáutica, que para esta región aérea el sistema ADS-B consistirá en la radiodifusión por una aeronave de su posición (latitud y longitud), altitud, velocidad, identificación de la aeronave obtenida de los sistemas de abordo. Recomienda también el uso de sistema GMSS para obtener la posición basado en el GPS de la aeronave y aclara que 


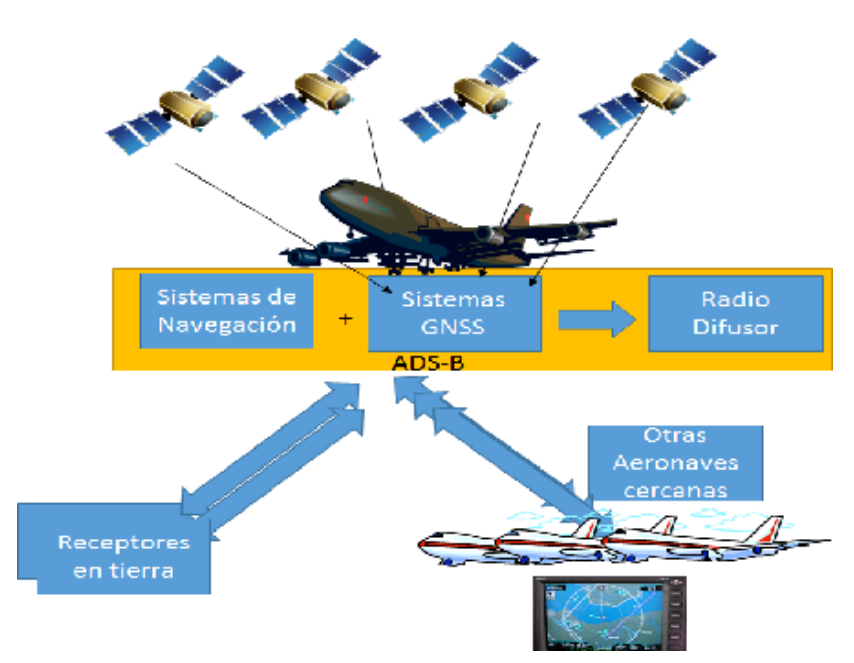

Figura 4. Esquema Sistema ADS-B.

Fuente: Elaboración del autor.

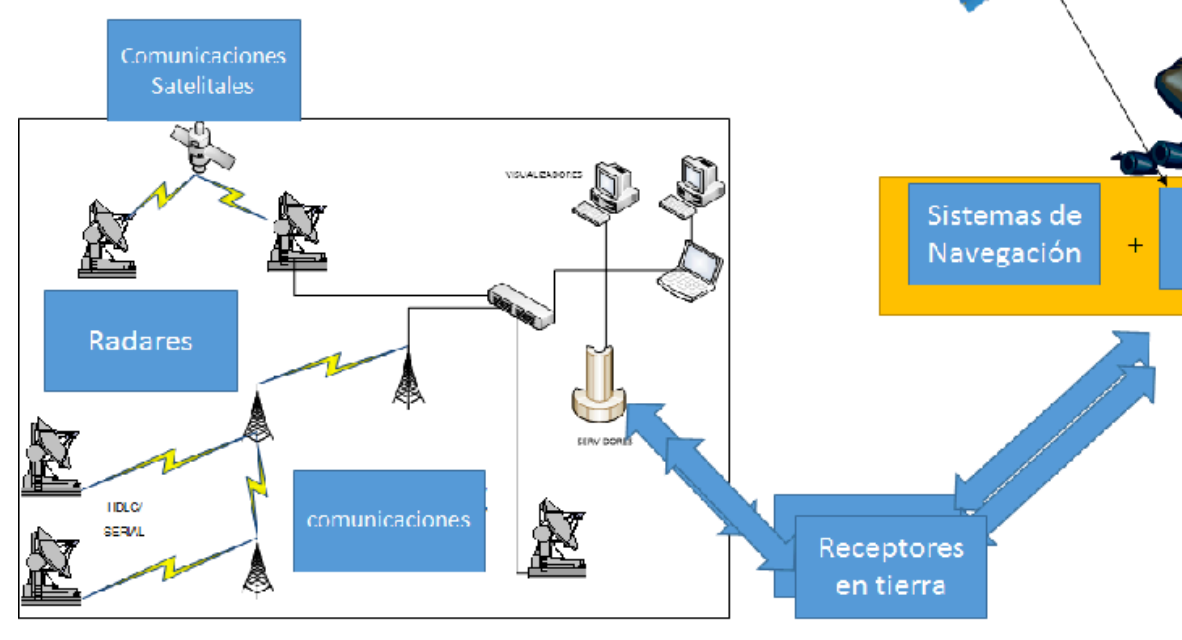

Figura 6. Esquema Sistema ADS-B integrado al sistema de vigilancia Colombiano.

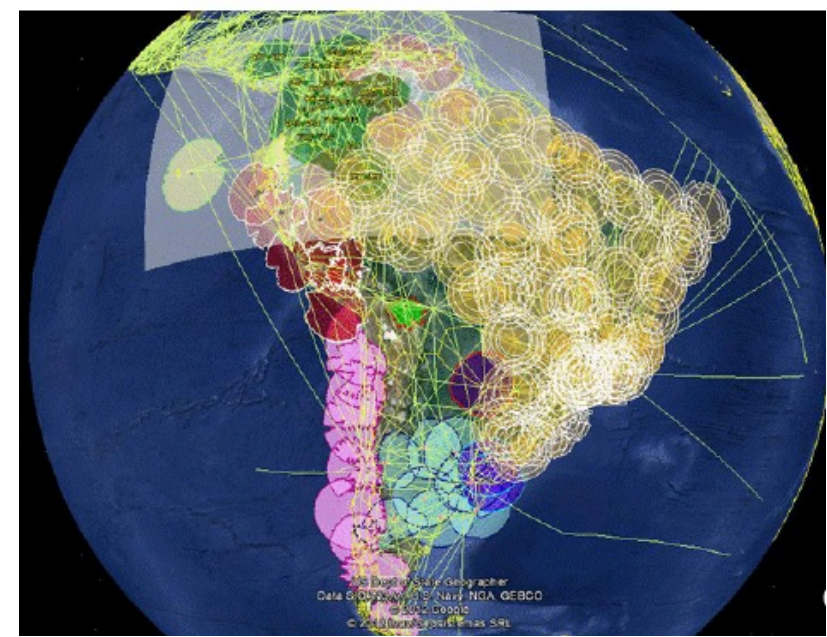

Figura 5. Cobertura sistemas vigilancia SAM.

Fuente: O. R. S. OACl, (2013). Guía de Consideraciones Técnicas Operacionales para la Implantación del ADS-B en la región SAM.

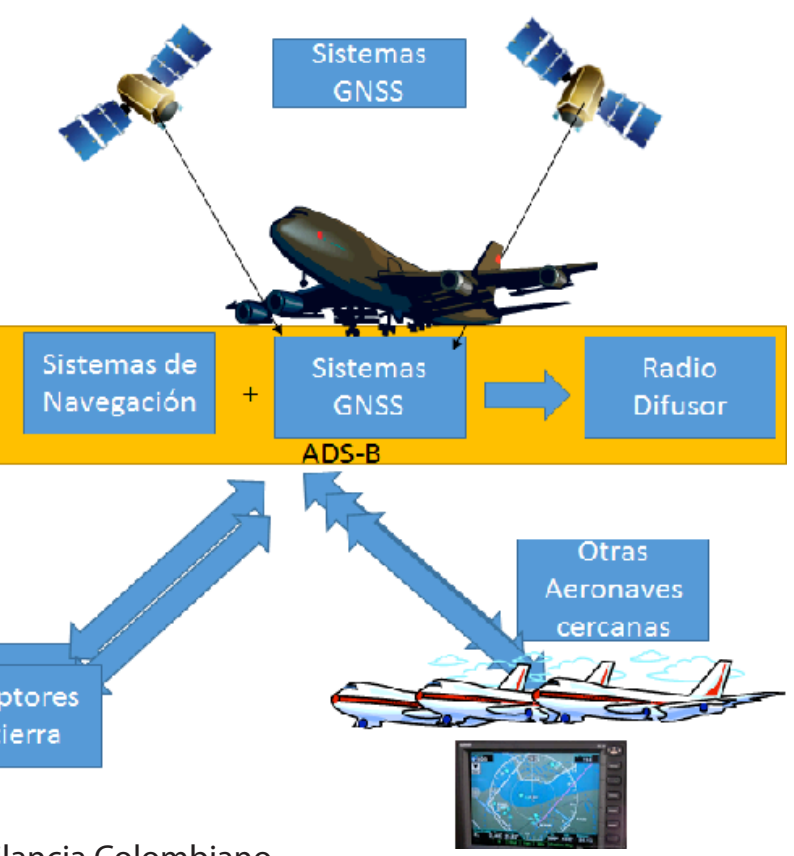

Fuente: elaboración del autor.

los sistemas inerciales de la aeronave por sí mismos no cuentan con la suficiente precisión, y que de ser utilizados deben enviar un mensaje de calidad no determinada o desconocida. En la Figuras 4 se muestra la estructura general del sistema.

En el documento también se explica los detalles técnicos para realizar la implementación del sistema en el país y se hace una descripción del estado de cobertura del sistema de vigilancia aeronáutica completo, teniendo en cuenta los planes de integración de ADS-B para la región de Sur América. En la Figura 5 se observan la cobertura radar y la cobertura ADS para la región. Debido a estas nuevas tendencias, se están realizando estudios sobre el tema, como los publicados en Flavio et al (2011); Schafer et al (2014); Bian y Moertl (2012); Flavio y Camargo (2011) que analizan los efectos en la reflexión atmosférica de las señales ADS, la precisión del sistema ADS-B, las aplicaciones realizadas en Europa y su cobertura, e incluso se publica un estudio en el que se analiza el desempeño del sistema en países como China.

Existe una clasificación de los ADS-B. el sistema Clase A soporta tanto las señales ADS-B OUT y ADS-B IN, 
los sistemas Clase B soportan solamente el envío de información radiodifundida (Mensajes ADS-B OUT) típicamente son utilizadas en vehículos de superficie u obstáculos fijos y los sistemas Clase $C$ sólo pueden recibir información(ADS-B IN).

Dentro de los sistemas de vigilancia, actualmente la tendencia es tener un sistema cooperativo de vigilancia aérea, en el que se integre toda la información disponible, más un componente radiodifundido que permita a las aeronaves mantener su propia conciencia situacional como se define en Ress (2009); de esta manera, el sistema propuesto se podría ver en la Figura 6 .

Como se aprecia, este tipo de sistemas cuentan con grandes ventajas, que con una adecuada integración y entendimiento de la tecnología podrían ser adecuadas a las condiciones colombianas y en especial a solucionar algunos problemas de la FAC, para el desarrollo de operaciones en áreas que no cuentan con suficientes ayudas aeronáuticas.

\section{Método / Inicio de la investigación}

Una de las misiones asignada por el comandante de la FAC al Centro de Desarrollo Tecnológico para la Defensa, CETAD, son los desarrollos de tecnologías estratégicas para el país. En los últimos años, el CETAD ha venido desarrollando una línea de investigación en Comando y Control donde el sistema HORUS ha sido su principal producto. Desde finales del 2012 se comenzó un análisis documental y bibliográfico que permitiera entender el modo de funcionamiento del sistema ADSB y del radar secundario, con el fin de integrar estos sistemas al proyecto HORUS (Comando y Control).

Posteriormente, se procedió a realizar un ejercicio basado en tecnologías Open Source. En el cual, utilizando un adaptador analógico de televisión USB para PC como el TV28T V2 o el USBDVB-T que cuenta con un chip Realtec y basado en los conceptos expresados en Frady (2004), donde se muestra cómo adaptar un hardware de bajo costo utilizando software adecuado se puede hacer un analizador de señal, donde la señal de RF es digitalizada, procesada y analizada a través del transductor que para este caso es el adaptador de TV para el PC.

La frecuencia de interés es de $1090 \mathrm{MHz}$, la cual se montó en un laboratorio que permitió la captura de los datos en esta banda de frecuencia, su decodificación y por último la graficación en tiempo real de los datos pertenecientes a aeronaves próximas al aeropuerto de Rionegro que transmitían mensajes ADS-B radiodifundidos como se muestra en la Figura 7.

Con el desarrollo de este ejercicio y utilizando hardware genérico se inició el desarrollo de software propio del CETAD que permitiera realizar la misma tarea, con el fin de lograr un mayor entendimiento de la naturaleza de las señales. Para esto el primer impedimento fue la interpretación de la información (Ver Figura 8).

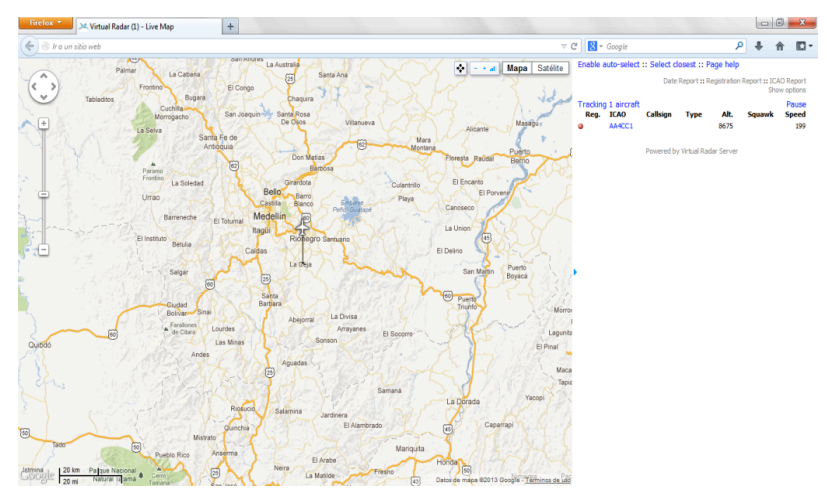

Figura 7. Captura de Datos ADS-B en RNG.

Fuente: Tomada de documentación del CETAD.

\begin{tabular}{|l|l|l|}
\hline ADS-B hexa: Bloc de notas & G & ES \\
\hline Archivo Edición Formato Ver Ayuda & \\
\hline 8CE8042B381D80758B1B4E4665B4 \\
8CE8042B381D8075891B4E5DCD36 \\
8CE808E703A10FB0C8CCBB69A \\
8CE8042B381D80758B1B4E4665B4 \\
8CE8042B381D80758B1B4E4665B4 \\
8CE8042B381D842F50C867B12E60 \\
8CE8042B381D842F50C867B12E60 \\
8CE8042B381D842F50C867B12E60 \\
8CE8042B381D842F50C867B12E60 \\
8CE8042B381D80758B1B4E4665B4 \\
8CE8042B381D842F50C867B12E60 \\
8CE808F381D843EA3219EC5730 \\
8CE80456703B085E9590D632514 \\
9702390711F1A78CD \\
8CE8042B381D80757F1B577694CB \\
\hline
\end{tabular}

Figura 8. Datos capturados sin interpretar.

Fuente: elaboración del autor.

Después de un proceso de análisis de la trama capturada del ADS-B y posterior a analizar variantes de la forma como se interpreta la señal, se encontró la manera correcta de realizar esta decodificación, e integrarla en primera instancia con los desarrollos de sistemas de graficación que se han realizado en el Centro.

Para ello, antes de iniciar la decodificación del dato, es necesario determinar qué tipo de mensaje se está re- 
cibiendo en el enlace. En la Tabla 1 se muestran algunos de los tipos básicos de mensaje.

La identificación de cada tipo de mensaje se logra a través de la interpretación de los primeros 5 bits del datagrama, los cuales se identifican en la Tabla 1.

Dentro de las principales características del sistema ADS-B resalta el hecho que siempre está enviando la información a posibles receptores en tierra o en aire.

Tabla 1. Mensajes ADS-B

\begin{tabular}{|c|c|c|}
\hline $\begin{array}{l}\text { Número de } \\
\text { formato }\end{array}$ & Mensaje & Caracterización \\
\hline 0 & 00000 & $\begin{array}{c}\text { Vigilancia corta aire-aire } \\
\text { (ACAS) }\end{array}$ \\
\hline 4 & 00100 & $\begin{array}{l}\text { Vigilancia, petición de } \\
\text { altitud }\end{array}$ \\
\hline 5 & 00101 & $\begin{array}{l}\text { Vigilancia, petición de } \\
\text { identidad }\end{array}$ \\
\hline 11 & 01011 & $\begin{array}{c}\text { Llamada general en Modo } \\
\text { S solamente }\end{array}$ \\
\hline 16 & 10000 & $\begin{array}{c}\text { Vigilancia larga aire-aire } \\
\text { (ACAS) }\end{array}$ \\
\hline 20 & 10100 & Com-A, petición de altitud \\
\hline 21 & 10101 & $\begin{array}{l}\text { Com-A, petición de } \\
\text { identidad }\end{array}$ \\
\hline
\end{tabular}

La estructura de la trama ADS-B en el formato general descendente es:

\begin{tabular}{|l|l|l|l|l|l|}
\hline 1 & \multicolumn{2}{|c}{6} & \multicolumn{2}{c}{9} & \multicolumn{2}{c}{33} & \multicolumn{2}{c|}{89} \\
\hline DF & & CA & AA & ME & PI \\
\hline
\end{tabular}

Figura 9. Trama ADS-B.

Fuente: Documentación del CETAD.

El formato utilizado para las señales espontáneas ampliadas ADS de enlace descendente de 112 bits tiene como identificador DF $=17$ y costa de los siguientes campos:

DF Formato de enlace descendente.

CA Capacidad.

AA Dirección anunciada.

PI Paridad/identificador de interrogador.

Dentro de los campos más importantes de la trama para el CETAD se encuentran en el campo ME y son:
- Posición de la aeronave: contiene la latitud, longitud y altitud.

- Velocidad de vuelo: contiene la velocidad y el rumbo de la aeronave.

- Mensaje de ID y de la categoría del avión: contiene el identificador de vuelo y el tipo de aeronave.

$$
T=0 \quad \text { LATITUD }=14845
$$

001110001110111101000,00001110011111110110001101100011100 $\mathrm{TC}=8 \quad \mathrm{P}=0$ ALTITUD $=3828 \quad \mathrm{~F}=0$ LONGITUD $=36238$

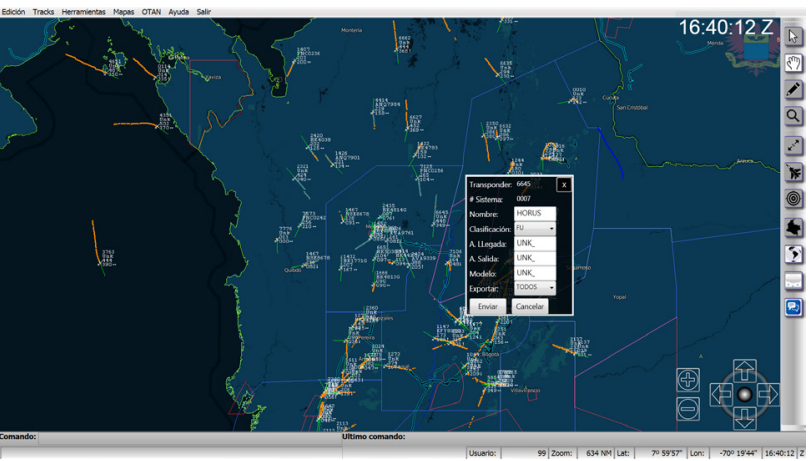

Figura 10. Graficación de ADS-B en Software FAC.

Fuente: Documentación Sistema Horus-ADS.

Posterior a que se lograra en el CETAD, la graficación con hardware de bajo costo y software open source, se inició el proceso de programación de un algoritmo propio que permitiera la graficación de las señales proveniente de equipos ADS-B. Aprovechando la cercanía del aeropuerto José María Córdoba se inició el experimento y se logró como se muestra en la Figura 10 que los tráficos próximos al área del experimento, fueran graficados en tiempo real, independiente de la cobertura radar. Lo anterior, permite comenzar un proceso de análisis de la información y de las posibles aplicaciones que tendría esta tecnología para la FAC.

Se decide entonces iniciar adquisición de hardware de más sensibilidad y más potencia, con el que se pretende ampliar el radio de alcance del sensor, buscando una aplicación más precisa y con beneficios potenciales para suplir deficiencias en los sistemas de control militar aéreo para la institución.

Se empieza entonces a trabajar con un URSP N200/210 producido por National Instruments, que básicamente es una serie de FPGAS ${ }^{4}$ configurables y pro-

4 FPGA del inglés Field Programmable Gate Array, es un dispositivo que cosiste en bloques de lógicos cuya interconexión y funcionalidad puede ser programada. 
gramables con diferentes anchos de banda y diferentes parámetros de radio Frecuencia. Para el caso se desarrolló un software y se hizo la configuración del hardware para trabajar en la banda objetivo (1090 Mhz). La Figura 11 muestra algunas capturas de los desarrollos que se hicieron utilizando Labview, software de programación de National Instruments.

Gracias a estos desarrollos, el CETAD ha logrado comprender la información enviada y procesada por los sensores ADS-B, en mensajes largos y cortos y se espera que con la adquisición de mejores sensores se pueda cubrir un área objetivo mucho mayor. Inicialmente en el desarrollo y por la similitud de la naturaleza de la señal se planteó la posibilidad que el radar secundario de los sensores de vigilancia y la tecnología ADS-B compartieran formatos de modulación y datagramas.
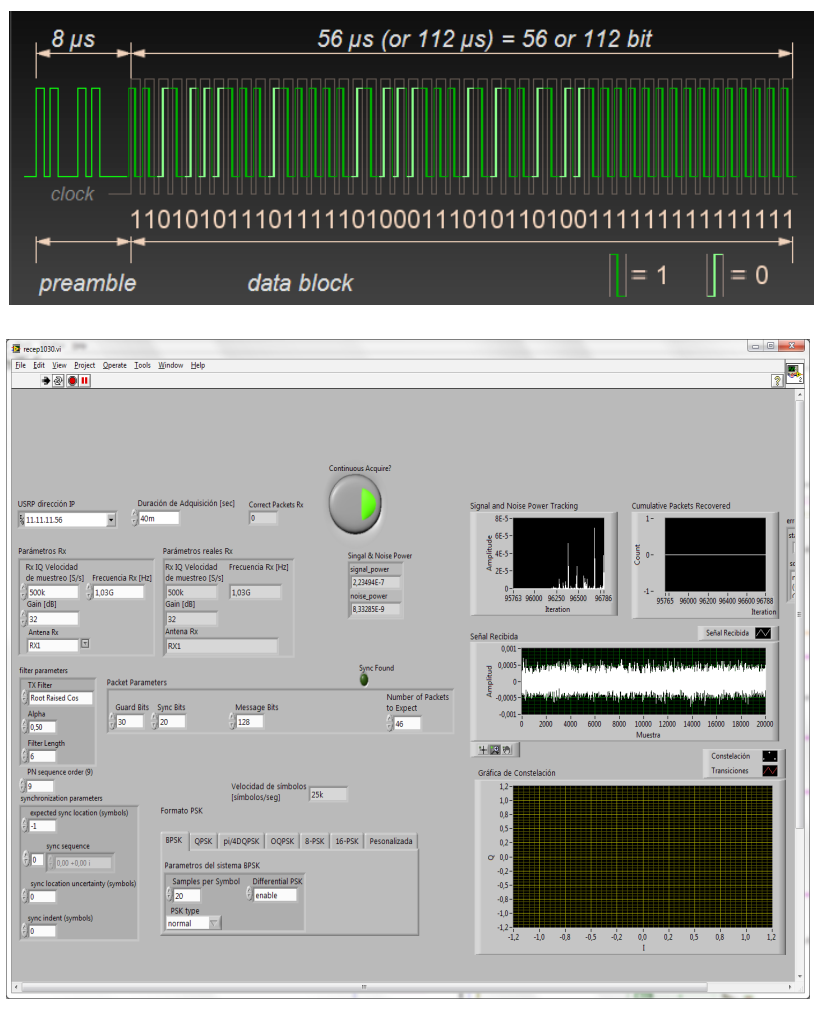

Figura 11. Analizador de señales ADS-B desarrollado en el CETAD.

Fuente: Documentación del CETAD.

Este fue uno de los primeros retos en los que se centró la investigación y después de varios experimentos y de revisar la bibliografía disponible, se ha concluido que aunque ambos servicios están ubicados en la banda de $1090 \mathrm{MHz}$, ambos son independientes y son tipos de modulación completamente diferente.

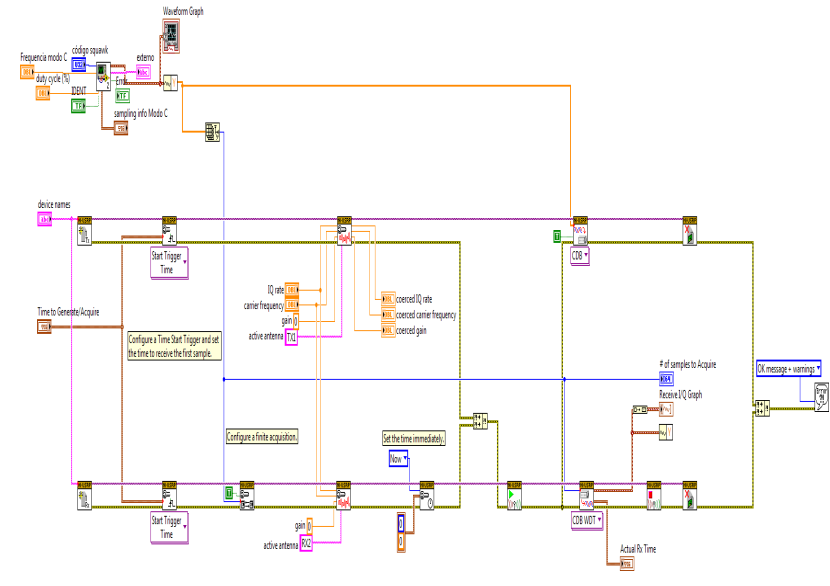

Figura 12. Software desarrollado por CETAD para procesamiento ADS-B.

Fuente: Documentación del CETAD.

Esto ha motivado que el CETAD también inicie el análisis del radar secundario, con el fin de dominar esta área y poder desarrollar tecnologías derivadas, apropiadas para la FAC.

La importancia de la tecnología ADS-B es tanta en el país que para finales del 2014, la UAEAC estará integrando un importante número de receptores en tierra a su sistema CNS/ATM que pretenden completar el esquema del sistema de vigilancia civil. Por su parte, la FAC también está evaluando la posibilidad de interpretar las categorías de euro control propias del ADS-B en su nuevo sistema de comando y control para el 2015 (Ver Figura 12).

\section{Conclusiones}

Para poder generar las señales ADS-B y modularlas para enviarlas al espectro, por medio del radio NI USRP N210, fue necesario desarrollar módulos en labview y contar con hardware tipo RX/TX con bandas desde 400 $\mathrm{MHz}$ hasta $2 \mathrm{GHz}$.

Aunque existen muchas similitudes entre el sistema de radar secundario y el sistema ADS-B, son sistemas completamente diferentes tanto desde su concepción como en su estructura técnica. Sin embargo, mantienen filosofías y estructuras de trabajo complementarias.

Los radares secundarios necesitan de una señal de interrogación para poder responder.

Los Sistemas ADS-B y los radares en Modo S han brindado soluciones a los principales errores presentados por los radares convencionales, gracias a la implementación de un pequeño enlace de datos logran la 
eliminación de problemas como el garbling, y dan la garantía de poder identificar cada aerodino.

Tanto los ADS-B como los transpondedores modo $S$ transmiten señales espontáneas para apoyar la radiodifusión de la posición obtenida de la aeronave, lo que permite la compatibilidad de las redes ADS-B y modo $\mathrm{S}$.

Como los radares secundarios tienen varios modos de interrogación A, C, Intermodo y S, si un radar está configurado en modo $S$, se podría pensar en recibir información en un ADS-B IN desde un respondedor modo-S.

El sistema ADS-B brinda más información relativa al espacio aéreo que otros sistemas, para que los pilotos puedan volar a distancias seguras de otras aeronaves sin necesidad de depender de los controles de tráfico aéreo.

Las ventajas más importantes del sistema ADS-B son la capacidad de vigilancia aire-aire. Es decir, entre aviones, capacidad de vigilancia en zonas que no tienen cobertura radar, brindan información del tráfico al piloto en tiempo real, permiten predecir con mayor exactitud los tiempos de vuelo.

Los sistemas ADS-B podrían convertirse en un sistema alternativo de control en aeródromos militares o zonas en las que se realizan operaciones militares aéreas, elevando los índices de seguridad aérea y facilitando el control tanto en aproximación como en superficie.

\section{Referencias}

Bian, B., and Moertl, P. M. (2012). Global positioning system accuracy under varying ionospheric conditions for surface Automatic Dependent Surveillance-Broadcast applications. In 12th Integrated Communications, Navigation and Surveillance Conference: Bridging CNS and ATM, ICNS 2012, pp. A31-A318.

Chiang, K. W., and Huang, Y. W. (January, 2008). An intelligent navigator for seamless INS/GPS integrated land vehicle navigation applications. Appl. Soft Comput., 8 (1), pp. 722-733.

Flavio Vismari, L., and Camargo Junior, J. B. (July, 2011). A safety assessment methodology applied to CNS/ATM-based air traffic control system, Reliab. Eng. Syst. Saf., 96 (7), pp. 727-738.

Flavio Vismari L., and Camargo Junior, J. B. (July, 2011). A safety assessment methodology applied to CNS/ATM-based air traffic control system. Reliab. Eng. Syst. Saf., 96, (7), pp. 727-738.

Frady, S. (WNC IEEE) (March, 2014). Applications for RTL based Software Defined Radios. [Online]. Available: http://sites.ieee. org/wnc/files/2014/04/IEEE_Frady_SDR_Mar_2014.pdf.

Harmonisation, E. A. T. C., and Programme, A. I., (August, 2002). Eurocontrol Standard Document For Part 2a Transmission of Monoradar Data Target Reports.
Harrison, M. J. (2006). ADS-X the Next Gen Approach for the Next Generation Air Transportation System. ieee/aiaa 25TH Digit. Avion. Syst. Conf.

International Civil Aviation Organization (ICAO). (2012). Manual on Airborne Surveillance Applications.

International Civil Aviation Organization (ICAO). (September, 2011). ADS-B Implementation And Operations Guidance Document.

Miquel, T.; Mora-Camino, F., and Loscos J. M. (2006). Path stretching and tracking for time-based aircraft spacing at meter fix. In Collection of Technical Papers - AlAA Guidance, Navigation, and Control Conference, 1, pp. 411-418.

Nolan, M. S. (1998). Fundamentals of Air Traffic Control. Third Edit. Organización de los Sistemas de Sensores de Vigilancia (OACl). (1998). Manual sobre ensayo de sistemas del radar de vigilancia. Primera Ed.

Organización de Aviación Civil Internacional. (2006). Anexo 10. Telecomunicaciones aeronáutica.

O. R. S. OACI. (2013). Guía de Consideraciones Técnicas Operacionales para la Implantación del ADS-B en la región SAM.

Rees, M. (Head of CNS-EUROCONTROL) (2009). The Eurocontrol Surveillance Strategy. In ENRI International Workshop on ATM/CNS.

Rushby, J. (1994). Critical System Properties, 43 (2), pp. 189-219.

Schafer, M.; Strohmeier, M; Lenders, V.; Martinovic, I., and Wilhelm, M. (2014). Demonstration abstract: OpenSky A large-scale ADS-B sensor network for research. In IPSN-14 Proceedings of the 13th International Symposium on Information Processing in Sensor Networks, pp. 313-314.

Skolnik, M. I. (1990). Radar Handbook. Second Ed. McGraw-Hill.

Strohmeier, M.; Schafer, M.; Lenders, V., and Martinovic, I. (May, 2014). Realities and challenges of nextgen air traffic management: the case of ADS-B. IEEE Commun. Mag., 52 (5), pp. $111-118$.

Tomlin, C. (1998). Hybrid Control of Air Traffic Management System [PhD Dissertation]. California University.

Yamamoto, K. (2014). Air Traffic Management and Systems, vol. 290, pp. 3-14. Tokyo: Springer Japan.

Zhang J., Liu, W., and Zhu, Y. (August, 2011). Study of ADS-B Data Evaluation. Chinese J. Aeronaut, 24 (4), pp. 461-466.

Para citar este artículo: Flórez-Zuluaga, J. (2014). Apropiación y uso tecnologías ADS-B en el CETAD. Ciencia y Poder Aéreo, Vol. 9 (1). Pp. 125-134 